\title{
NGAL is a potential early diagnostic and prognostic biomarker of severe acute pancreatitis
}

E arly diagnosis of severe acute pancreatitis may be facilitated and patient outcomes improved by measuring serum levels of neutrophil gelatinase-associated lipocalin (NGAL). "We have identified a new, early biomarker [NGAL] that can help solve the clinical dilemma of whether a patient with symptoms suggestive of acute pancreatitis will develop mild or severe (life-

threatening) disease," says Surinder Batra, who works at the University of Nebraska Medical Center.

Following their previous observation that NGAL levels were markedly elevated in patients with pancreatitis but not in healthy controls, Batra and colleagues decided to investigate whether NGAL had diagnostic potential as a biomarker for acute pancreatitis and could discriminate mild acute pancreatitis from severe acute pancreatitis.

To study the rise in NGAL levels over time, the researchers used a mouse model of pancreatitis-infusion of taurocholate into the mouse common bile duct induced an episode of severe acute pancreatitis, whereas infusion of saline induced an episode of mild acute pancreatitis. Intraperitoneal injection of saline was used as a control.

Mouse serum NGAL levels, measured by using a sandwich enzyme linked immunosorbent assay (ELISA), rose within $6 \mathrm{~h}$ of induction of either form of acute pancreatitis. In the model of severe acute pancreatitis, serum NGAL levels were significantly elevated even $24 \mathrm{~h}$ after pancreatitis ensued. By contrast, serum NGAL levels had begun to decrease by the same time point in the model of mild acute pancreatitis. "These results clearly demonstrate that the rise of NGAL in serum is an early marker of the severity of acute pancreatitis," clarifies Batra.

Serum samples were also taken from 33 healthy individuals, 28 patients with chronic pancreatitis and 44 patients with acute pancreatitis ( 28 patients with
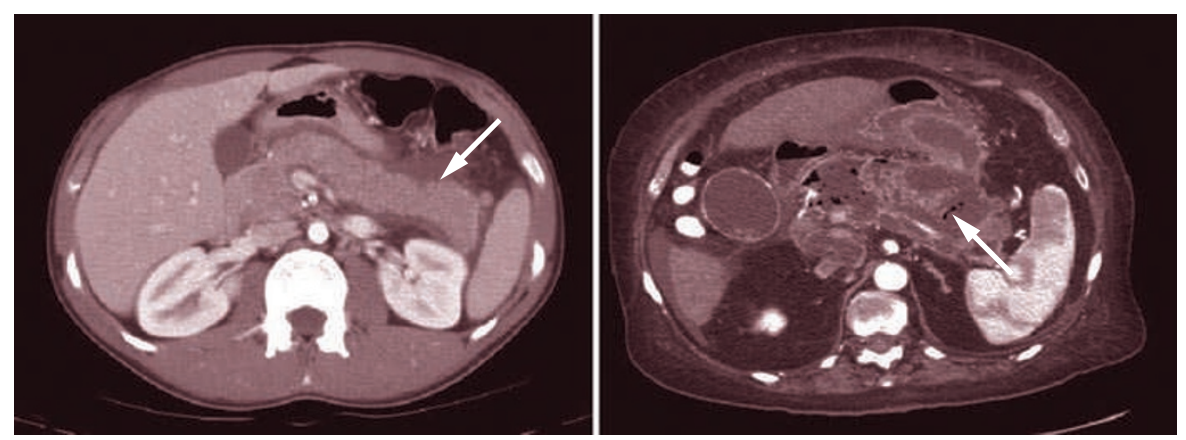

CT scans: mild acute pancreatitis (left) showing normal enhancement of the pancreas after intravenous contrast with peripancreatic stranding (arrow); severe acute pancreatitis (right) showing necrosis involving the whole of the pancreas with gas (arrow) in the necrotic collection. Courtesy of G. I. Papachristou.

mild acute pancreatitis and 16 with severe acute pancreatitis) at a defined time after admission to hospital. The level of NGAL present was again quantified by a sandwich ELISA. Serum C-reactive protein levels were also measured and the APACHE II score (calculated on admission and on days 1 , 2, 3, 4 and 7 after admission) and Ranson's score (calculated $48 \mathrm{~h}$ after admission) recorded.

Serum NGAL levels were elevated in patients with acute pancreatitis, but not in healthy individuals or patients with chronic pancreatitis. A rise in NGAL levels was found only in those patients who had severe acute pancreatitis but not mild acute pancreatitis and that rise occurred early-within $48 \mathrm{~h}$ (equivalent to the rise in serum NGAL levels within $6 \mathrm{~h}$ in the mouse model of acute pancreatitis).

NGAL levels were also found to be significantly higher in patients with severe acute pancreatitis who died versus those who lived. "This suggests that the measurement of NGAL levels could be useful to predict the outcome in those patients with acute pancreatitis who are severely ill," explains Batra. In addition, patients with severe acute pancreatitis who developed multi-organ failure had significantly higher serum NGAL levels than those who did not develop multiorgan failure-the researchers believe that measuring NGAL levels could, therefore, also predict the onset of this known complication of severe acute pancreatitis.

Also of note, albeit in a limited sample set, serum NGAL levels performed better than the clinical scoring systems as a predictor of patient outcome.

The researchers now plan to investigate the mechanisms by which NGAL is upregulated in acute pancreatitis and more specifically in severe acute pancreatitis. As their study was conducted using samples from a relatively small number of patients from a single center, they also intend to validate the findings of this pilot study in a larger number of patients. Another plan is to take serial measurements of NGAL in patients for the duration of their hospital stay.

"If validated in a larger study, NGAL could potentially circumvent complex clinical scoring systems and offer a more sensitive and early indication of patient outcome," concludes Batra. "This knowledge can be used to introduce appropriately aggressive measures much before clinical signs of severe disease set in, thus potentially saving more lives."

Natalie J. Wood

Original article Chakroborty, S. et al. Elevated serum neutrophil gelatinase-associated lipcalin is an early predictor of severity and outcome in acute pancreatitis. Am. J. Gastroenterol. 105, 2050-2059 (2010) 